\title{
Responsabilidad Social Universitaria RSU. Análisis de stakeholders. Facultad Ciencias Administrativas Universidad de Guayaquil
}

\author{
Víctor Hugo Briones Kusactay* \\ Rafael EMiliano Apolinario Quintana** \\ Margarita Denisse Avila Pita*** \\ Ecuador
}

\section{Resumen}

El presente artículo da cuenta sobre la forma como los stakeholder de la FCA-UG; dan un aporte al desarrollo en temas de RSU que necesita Ecuador, siendo los estudiantes quienes tienen mayor percepción sobre la necesidad de involucrase con la comunidad para fomentar una cultura socialmente responsable.

\section{Introducción}

Ecuador está atravesando por un proceso de cambios estructurales en su ordenamiento jurídico interno, la Constitución de la República de

* Master en Administración de Empresas. Profesor titular de la Facultad de Ciencias Administrativas, Universidad de Guayaquil. Contacto: victor.brionesk@ug.edu.ec

** Master en Negocios Internacionales. Profesor titular de la Facultad de Ciencias Administrativas, Universidad de Guayaquil. Contacto: rafael.apolinarioqu@ug.edu.ec

**:Ingeniera en Comercio Exterior, Universidad de Guayaquil. Contacto: m. denissavila@gmail.com 
2008 contempla reformas en los poderes del Estado, definiéndolos el Ejecutivo, Legislativo, Judicial, Electoral y de Participación Ciudadana y control Social, por lo tanto, se aprecian avances significativos en términos de Responsabilidad Social. La nueva Ley Orgánica de Educación Superior LoEs, en concordancia con la Constitución de la República, contempla el nuevo rol de las universidades, estas tienen entre sus ejes la Academia, Investigación, Vinculación y Gestión, lo cual demuestra el rol social de la universidad que debe de coordinar su trabajo con los órganos de gobierno y sectores productivos, tendiendo puentes de innovación, ciencia y tecnología que aportan ventajas competitivas al servicio y desarrollo del país. Este nuevo despertar de la conciencia social es la fuerza impulsora que mueve a la Universidad de Guayaquil a trabajar en el desarrollo de las zonas 5 y 8 del país asignadas a sus competencias por la Secretaría Nacional de Planificación y Desarrollo, Semplades, y que corresponden a las provincias de Galápagos, Santa Elena, Guayas, Los Ríos y Bolívar (zona 5); y, Guayaquil, Durán y Samborondón (zona 8).

De allí que la Facultad de Ciencias Administrativas de la Universidad de Guayaquil comienza a involucrarse en estos nuevos retos que exige la sociedad para su convivencia armónica y desarrollo equitativo, empezando a estudiar sus relaciones con los stakeholders. En consonancia con ello, la pregunta de investigación del presente estudio plantea cómo los stakeholders de la FCA-UG, dan un aporte al desarrollo en temas de RSU que necesita Ecuador, cuyo objetivo consiste en analizar la valoración de las estrategias en Responsabilidad Social que tienen los stakeholders de la Universidad de Guayaquil en la Facultad de Ciencias Administrativas.

\section{Revisión literaria de la vinculación de la universidad con la sociedad y el Estado}

Según Mora (2012), la Rsu es la capacidad de gestión y vinculación con la comunidad que debe poseer un ente de educación superior, lo cual indica que el término sociedad debe abarcar no solo lo que se conoce como sociedad civil, sino hay que pensarlo como un sistema complejo 
donde interactúan también las empresas y el Estado. La gestión educativa de la RSU operará con indicadores cualitativos y cuantitativos que permitan el desarrollo de programas inclusivos (Martins, Claudeville, y Parada, 2013). La relación que mantiene la universidad con la sociedad puede ayudar a mejorar el desarrollo de un país, analizando y aportando con los conocimientos frente a las problemáticas actuales (Martínez, 2010). Todas las organizaciones generan impactos en el plano organizacional (dimensión interna de la Rs) y social (dimensión externa de la Rs), además de los propios de su tipo organizacional (Gorrochategui, 2012).

En la actualidad, las universidades deben desempeñar un papel que permita a los estudiantes vincularse con la sociedad y no solo formarse como profesionales (Vallaeys, 2008). En América Latina las universidades han evolucionado dejando a un lado su rol como institución formadora, para convertirse en una herramienta de preparación en la que los estudiantes compiten por un empleo (Gasca y Olvera, 2011). Un grupo de universidades de Latinoamérica y el área andina ejecutan programas socialmente responsables. Sin embargo, no han asumido la RSU como un modelo de gestión (Jácome, Viteri y Medina, 2013). Las universidades deben cambiar sus modelos educativos y administrativos para poder comprometerse socialmente, de lo contrario, no se estaría aplicando modelos de RSU (Vallaeys, 2010). Las instituciones de educación superior deben responder a las necesidades sociales incorporando dos formas para manifestarse. La primera, a través de la imagen que transmite la universidad y se proyecta a su entorno. La segunda, en la formación de capacidades y valores de los estudiantes (Jácome y Viteri, 2012).

La forma más acertada para transformar una universidad es aplicando herramientas que permitan la gestión de responsabilidad, no solo en ámbitos de intervención con la sociedad, sino con prácticas medioambientales, complementando su evolución a través de normas tales como Iso 26000, AA 1000, SA 8000 . Estas tienen como objetivo convertir a las organizaciones en entidades socialmente responsables (Vallaeys, 2013).

La aplicación de la RSU debe ser discreta, paciente y eficaz, en coherencia con lo que está haciendo como universidad y lo que se espera 
como sociedad (Vallaeys, 2010). El sistema educativo debe estar especializado no solo en informar, sino también en formar estudiantes. Un estudiante formado asimila los cambios, los acepta y contribuye a la renovación y progreso de la sociedad (García, 2008).

En Ecuador, la nueva reforma al régimen académico universitario califica a nivel nacional a todas las universidades (CEAACES, 2013), otorgando a cada una un rango en posición académica.

En la actualidad todas las universidades del Ecuador deben aplicar correctamente la norma Iso 26000 (Responsabilidad Social), la cual indica que las universidades deben de estar vinculadas a la participación Activa y el Desarrollo con la Comunidad (García, 2011). La educación superior debe contribuir al pensamiento crítico sobre los problemas éticos en el ejercicio de las distintas disciplinas (Gilli, Schulman, y García, 2016).

En consonancia con ello, universidades como la Escuela Superior Politécnica del Litoral realizan eventos alrededor de su programa de vínculos con la colectividad, para que sus estudiantes desarrollen la implementación de la RSU en su entorno. Otra de las universidades que a nivel nacional está aplicando la Rsu, en consideración de los stakeholders, es la Universidad Casa Grande. Esta ha realizado diversos eventos y estudios, asumiendo un esquema organizacional programado a futuro, con una vinculación de la parte interna y externa que contribuya al bienestar de la universidad y la sociedad.

Para Calle (2012), es importante hacer énfasis en la nueva reforma al Régimen Académico, la Ley Orgánica de Educación Superior, la cual trata sobre las instituciones y la colectividad; específicamente en su artículo 3 indica que las instituciones del Sistema Nacional de Educación Superior tienen como misión la búsqueda de la verdad, el desarrollo de las culturas: universal y ancestral ecuatoriana, de la ciencia y la tecnología, mediante la docencia, la investigación, la gestión y la vinculación con la colectividad (Hernán Cortés, 2012).

En consonancia con ello, el objetivo de este estudio es analizar la valoración de las estrategias en Responsabilidad Social que tienen los stakeholders de la Universidad de Guayaquil en la Facultad de Ciencias Administrativas. Respecto a la aplicación de la RSU, Martínez (2009) menciona que Colombia es uno de los países que en que las 
universidades son el ente social primordial para el desarrollo de la RSU debido a su naturaleza y constitución.

El término universitas, surgido en la baja Edad Media para referirse a la agremiación de alumnos y maestros, significa en sentido primario una asociación, un gremio o una sociedad, hace referencia al concepto de conglomerado, de unidad de integración. De igual manera, otro autor considera que a través de propuestas concretas busquen enfocar la educación universitaria hacia la responsabilidad social, haciendo transparente la gestión social de estos establecimientos (Ureta, 2007).

La RSU es un término que no admite parcialidades ni segmentaciones: no se pueden desarrollar actuaciones responsables en un ámbito y dejar otros ocultos a la mirada ética. Por lo tanto, no se trata solo de crear una nueva oficina de RSU o de mejorar el área de extensión y la proyección social solidaria de la universidad sin tocar los demás procesos de la institución (Vallaeys, Cruz y Sasia, 2009). La Rsu planifica de una manera estratégica cuáles son los elementos a incorporar para desarrollar una cultura Socialmente Responsable entre las universidades y la sociedad, para esto se identifican 6 elementos referentes a la RSU, los cuales se mencionan a continuación:

Asociarse con otras instituciones: Realizar proyectos de forma conjunta, buscando incluir a todos los sectores: público, privado y sociedad civil.

Rendición de cuentas: Lograr la difusión sobre la responsabilidad social de las organizaciones para lograr construir ciudadanos informados.

Gestión de impactos: Analiza el contexto social donde se desenvuelve la organización.

Asociar a todas las partes afectadas: Detectar los problemas y buscan soluciones en las que participan los stakeholders. Reportes de diagnósticos regulares: Muestran determinados indicadores que permiten detectar impactos negativos y minimizarlos.

Reportes de sostenibilidad: Se muestra información económica-financiera social y ambiental de la organización (relación con los stakeholders) (Gasca y Olvera, 2011). 
Sin embargo, en la concepción de la RSU, a partir de los 6 impactos universitarios, el más importante de estos, el fundamental, el que conduce a todos los demás, es el impacto cognitivo. Mientras dejemos a los modelos mentales, epistemológicos y cognitivos promovidos por la universidad fuera del debate sobre el compromiso social de esta institución no se avanzaría en nada (Vallaeys, 2013). De acuerdo con Vallaeys et al., (2009), son cuatro los pasos imprescindibles de dar para llegar a la Responsabilidad Social. Ellos son: 1) compromiso, 2) autodiagnóstico, 3) cumplimiento, 4) rendición de cuentas.

No obstante, Pérez (2009) considera que la determinación de los impactos universitarios permite identificar las partes interesadas que deberán ser llamadas a participar en una gestión socialmente RSU: las autoridades universitarias, el personal (administrativo, docente e investigador) y los proveedores, desde la perspectiva del impacto organizacional, los estudiantes, desde la perspectiva del impacto educativo los investigadores y docentes, desde la perspectiva del impacto cognitivo, la comunidad local, la sociedad civil y los sectores público y privado como potenciales actores externos, desde la perspectiva del impacto social.

\section{Análisis metodológico}

Se realizó una cuadratura de información de cuatro sectores: autoridades, profesores, estudiantes y personal administrativo, mediante encuestas con preguntas dirigidas al conocimiento y aplicación de la RSU en la Facultad de Ciencias Administrativas de la Universidad de Guayaquil.

Se utilizó el método aleatorio simple cualitativo y se escogió el tamaño de la muestra de la población de los cuatro sectores involucrado en la investigación. 
Tabla 1. Mediana de los sectores involucrados

\begin{tabular}{|l|l|l|l|}
\hline Sectores involucrados & \multicolumn{1}{|c|}{ Población } & \multicolumn{1}{|c|}{$\begin{array}{c}\text { Tamaño de la } \\
\text { muestra }\end{array}$} & \multicolumn{1}{|c|}{ Muestra } \\
\hline Estudiantes & 12880 & $\begin{array}{l}\mathrm{N} \\
1+\left[(\% \mathrm{Ea})^{2} \mathrm{X} \mathrm{N}\right]\end{array}$ & 400 \\
\hline Docentes & 550 & & 230 \\
\hline P. Administrativos & 120 & & 120 \\
\hline Autoridades & 15 & & 15 \\
\hline
\end{tabular}

Fuente: encuesta a los stakeholders de la FCA-UG. Elaboración propia.

La población total de los sectores involucrados es de 13.565. Tomando una muestra finita, nos da como resultado 965 personas encuestadas. Cabe mencionar que respecto al personal administrativo y a las autoridades se consideró su población total debido al valor fundamental para reconocer y afrontar la verdadera Responsabilidad Social Universitaria frente a los docentes y estudiantes.

Cada encuestado contestó 20 preguntas, cada una de estas tiene una valoración de 5 puntos en la escala de Likert, por lo cual la suma de los resultados nos sirve como un indicador de la percepción de cada tipo de encuestado. La máxima puntuación daría 100 y la mínima 20.

Se usó el método de Kruskas Wallis para observar si las medianas entre las poblaciones se asemejan y así poder interpretar mejor la percepción que tiene cada tipo de encuestado.

Ho: Todas las medianas son iguales

Vs H1: Al menos una mediana difiere entre las demás

Tabla 2. Prueba de Kruskal-Walllis

\begin{tabular}{|l|l|l|l|l|}
\hline Tipo de Encuestado & \multicolumn{1}{|c|}{$\mathbf{N}$} & \multicolumn{1}{|c|}{ Mediana } & $\begin{array}{c}\text { Clasificación del } \\
\text { promedio }\end{array}$ & \multicolumn{1}{|c|}{$\mathbf{Z}$} \\
\hline Administración & 120 & 29,00 & 195,9 & $-10,10$ \\
\hline Autoridades & 15 & 29,00 & 260,0 & $-2,18$ \\
\hline Docentes & 230 & 30,00 & 260,1 & $-10,09$ \\
\hline Estudiantes & 400 & 41,00 & 514,4 & 17,22 \\
\hline General & 765 & & 383,00 & \\
\hline
\end{tabular}

Fuente: encuesta a los stakeholders de la FCA-UG. Elaboración propia. 
Dado que el valor $\mathrm{P}$ obtenido fue menor a 0,05 se procede a rechazar la hipótesis Nula, por lo tanto, se puede concluir que no existe suficiente evidencia estadística para afirmar que los estudiantes, autoridades, docentes y administradores tienen la misma percepción.

Usando el método de Mann Whitney para las posibles combinaciones de pares se observó que la percepción de los estudiantes es mayor que los docentes, autoridades y administradores. Al respecto, se realizó el siguiente análisis.

Ho: Mediana entre la percepción de Estudiantes es menor que de autoridades Vs

H1: Mediana entre la percepción de Estudiantes es mayor que de autoridades.

La estimación entre las medianas de Estudiantes con 41.00 y Autoridades 29. 00 es 11,000. Prueba de Estudiantes = Autoridades $v$ s. Estudiantes $>$ Autoridades es significativa en 0,0000, se procede a rechazar la hipótesis Nula

Ho: Mediana entre la percepción de Estudiantes es menor que de docentes Vs

H1: Mediana entre la percepción de Estudiantes es mayor que de docentes

La estimación entre las medianas de Autoridades 29.00 y Docentes 30.00 es significativa en 0,000 .

Prueba de Autoridades $=$ Docentes $v$ s. Autoridades $>$ Docentes. No se puede rechazar la hipótesis Nula, ya que W es < 1845,0

Ho: Mediana entre la percepción de autoridades es menor que de administradores Vs

H1: Mediana entre la percepción de autoridades es mayor que de administradores

La estimación de medianas entre Docentes 30.00 y Administradores 29.00 es 2,000. Prueba de Docentes $=$ Administradores vs. Docentes > Administradores es significativa en 0,0001

La prueba es significativa en 0,0000, Se rechaza la hipótesis Nula.

En la tabla 3 se encuentran resumidos los resultados de las comparaciones hechas previamente, aquí se puede notar de que la percepción 
entre los cuatro grupos es diferente. La que más se destaca entre todas es la de los estudiantes, ya que es mayor a las otras tres. La percepción de las autoridades es la menor de todas, mientras que la de los docentes es mayor a los administradores.

Tabla 3. Matriz de las percepciones de los diferentes tipos de encuestados

\begin{tabular}{|l|c|c|c|c|}
\hline & Estudiantes & Autoridades & Docentes & Administradores \\
\hline Estudiantes & $\mathrm{X}$ & $>$ & $>$ & $>$ \\
\hline Autoridades & $<$ & $\mathrm{x}$ & $<$ & $<$ \\
\hline Docentes & $<$ & $>$ & $\mathrm{X}$ & $>$ \\
\hline Administradores & $<$ & $>$ & $<$ & $\mathrm{X}$ \\
\hline
\end{tabular}

Fuente: encuesta a los stakeholders de la FCA-UG. Elaboración propia.

\section{Conclusión}

La universidad es un actor relevante para construir y anticipar escenarios alternativos encaminados a lograr una mayor equidad y cohesión social. El nivel de Responsabilidad Social dentro de la Universidad de Guayaquil en la Facultad de Ciencias Administrativas se evidencia en los resultados expuestos anteriormente, los cuales indican que los estudiantes tienen una mayor percepción acerca de la Responsabilidad Social. Sin embargo, la encuesta realizada puede no estar acorde con la realidad, ya que la mayoría de los estudiantes encuestados pueden tener sesgos en sus respuestas debido a su falta de madurez en la interpretación de la realidad social, o que han escuchado charlas de Rso por parte de sus Maestros autores de este artículo de RSU. Esto pudo incidir en los resultados de las encuestas, ya que el personal administrativo está desinformado al igual que un sector de Maestros y Autoridades.

La universidad debe ser transformada, romper paradigmas, adoptar nuevos modelos de desarrollo que integren, vinculen y articulen el sistema de Gobierno central, los sectores productivos y la sociedad en general.

Es evidente que aún falta implementar estrategias que nos permitan vincular la universidad con sus stakeholders, para que los estudiantes 
puedan concienciar sobre lo que sucede en su entorno y buscar soluciones a los problemas existentes. Es decir, tal y como se ha venido planteando, el concepto de responsabilidad social universitaria está en construcción permanente en virtud de la interacción que se establece entre la Facultad de Ciencias Administrativas y la sociedad, con miras a promover la cohesión social como fin primordial. Formación, investigación, liderazgo social y compromiso son los elementos sustantivos que determinan el formato de esta relación para hacer efectiva su incidencia en el desarrollo social, económico y productivo.

Se recomienda que para una próxima investigación la población estudiada sea más homogénea y contemple equidad de los actores, de tal forma que la muestra no contenga sesgos que nos lleven a errores de no aceptación de la Hipótesis Nula Ho, pensando que la Hipótesis Alternativa $\mathrm{H} 1$ es la que cumple los objetivos para el estudio sobre RSU en la Facultad de Ciencias Administrativas de la Universidad de Guayaquil. 


\section{Referencias}

Calle, I.M C. (2012). Universidad del Azuay. Recuperado de http://dspace. uazuay.edu.ec/bitstream/datos/2876/1/09328.pdf

Concejo de Evaluación, Acreditación y Aseguramiento de la Calidad de la Educación Superior. (2013). Evaluación de universidades 2013 - CEAACES. Recuperado de http://www.ceaaces.gob.ec/sitio/evaluacion-universidades-2013/

García, G. (2008). Responsabilidad social de las universidades De nuestra América. Recuperado de http://www.redalyc.org/articulo.oa?id=37303801

Gasca, E. y Olvera, J. (mayo-agosto, 2011). Construir ciudadanía desde las universidades, responsabilidad social universitaria y desafíos ante el siglo xxI. Convergencia, 18(56). Recuperado de http://www.scielo.org.mx/ scielo.php?pid=S1405-14352011000200002\&script=sci_arttext

Gilli, J., Schulman, D. y García, N. (2016). Temas de responsabilidad social en el currículo de tres universidades argentinas. En N. Gorrochategui, V. Martins de Oliveira, J. Domínguez Granda y H. Chero Valdivieso (comps.), Responsabilidad Social de las Organizaciones Rso Avances y propuestas en América Latina (pp. 293-331). Chimbote Perú: Universidad Católica Los Ángeles de Chimbote.

Gorrochategui, N. (2012). La extensión universitaria como expresión de la responsabilidad social universitaria (RSU). Caso argentino. En J. D.-C. Rama, La Responsabilidad Social Universitaria en la Educación a Distancia (pp. 77-101). Chimbote, Perú: Virtual Educa.

Hernán Cortés. (2012). Responsabilidad Social Universitaria. Una mirada a la Universidad Nacional de Colombia. Recuperado de http://www.bdigital.unal.edu.co/9022/1/822073.2012.pdf

Jácome, M., y Viteri, J. (2012). Índice integral para evaluar la responsabilidad social universitaria en Ecuador. Recuperado dehttp://scielo.sld.cu/scielo.php?script=sci_arttext\&pid=S1815-59362012000300009\&lang=pt

Jácome, M., Viteri, J., y Medina, A. (2013). Ingeniería Industrial. Modelo conceptual para la planificación estratégica con la incorporación de la responsabilidad social universitaria. Recuperado de http://scielo.sld.cu/ scielo.php?script=sci_arttext\&pid=S1815-59362013000100008\&lan$\mathrm{g}=\mathrm{pt}$

Martínez, R. (2009). Aplicaciones de Responsabilidad Social Universitaria en el contexto de formación en Colombia. Educación Superior y Sociedad, 
13(2), 163 - 174. Recuperado de http://ess.iesalc.unesco.org.ve/index. php/ess/article/viewArticle/44

Martins, F., Claudeville, M. M., y Parada, H. (junio, 2013). Gerencia Universitaria y Responsabilidad Social Universidad Simón Bolívar, sede Litoral. Revista de Investigación, 13(79). Recuperado de

http://www.scielo.org.ve/scielo.php?script=sci_arttext\&pid=S101029142013000200006

Mora, H. G. C. (2012). Responsabilidad Social Universitaria. Una mirada a la Universidad Nacional de Colombia. Recuperado de http://www.bdigital.unal.edu.co/9022/1/822073.2012.pdf

Pérez, F. (2009). Responsabilidad Social Universitaria. Recuperado de http:// www.uhu.es/consejo.social/pdf/documentos/responsabilidadsocialuniversita ria.pdf

Ricardo, F. G. (1 de Septiembre del 2011). Introducción a la Norma ISO26000: Guía sobre Responsabilidad Social. Recuperado de http://www. diarioresponsable.com/empresas/normas-y-acreditaciones/12551.html

Ureta, M. S. G. (2007). Responsabilidad Social y Universidad. Recuperado de http://www.techo.org/wp-content/uploads/2013/04/2007Gil.pdf

Vallaeys, F. (6 de octubre de 2008). Responsabilidad Social Universitaria. Recuperado de http://www.rsu.uninter.edu.mx/doc/marco_conceptual/PresentaciondelMarcoTeor icodelaRSU.pdf

Vallaeys, F. (2010). Ética y RSU. Recuperado dehttp://blog.pucp.edu.pe/blog/ eticarsu Vallaeys, F. (2013). 1. ${ }^{\text {er }}$ Congreso Internacional de Responsabilidad Social. Buenos Aires, Argentina. Recuperado de http://blog.pucp. edu.pe/media/410/20141123-arti_cirs_2013.pdf

Vallaeys, F., Cruz, C. de la, y Sasia, P. (2009). Responsabilidad Social Universitaria. Manual de primeros pasos.

Recuperado de http://idbdocs.iadb.org/wsdocs/getdocument. aspx?docnum $=35125786$ 